Aus der Inneren Abteilung des Katharinenhospitals Stuttgart (Direktor: Geh. Rat Sick).

\title{
Über sensorische Erscheinungen bei Tetanie und über Kombination der Tetanie mit andern Krampfneurosen. Von
}

\section{Felix Boenheim-Berlin.}

Wird ein Symptomenkomplex als nosologische Einheit erkannt, so werden anfangs naturgemäß nur voll ausgebildete, vorgeschrittene Krankheitsfälle diagnostiziert, besonders in einer Wissenschaft, die sich, wie die Neurologie, vorwiegend kasuistisch aufbaut. Erst einer späteren Zeit gelingt es, die Zugehörigkeit zu einer Krankheitsgruppe zu erkennen, bevor der einzelne Krankheitsfall voll entwickelt ist. Die Symptomatologie nimmt einen „proteusartigen" Charakter an. Die Prognose wird besser, die Therapie altiver.

Nach Beschreibung des klassischen Bildes der Tetanie wandte sich die Aufmerksamkeit selteneren Fällen zu. Zu den motorischen Symptomen gesellten sich sensible und (erst viel später) sensorische. Vestibulariserscheinungen, die sich nicht nur auf die (häufigen) subjektiven Klagen der Patienten beschränken, sind meines Wissens noch nicht mitgeteilt worden, abgesehen von einem Falle, der mit Menière kombiniert war. Es dürfte daher gerechtfertigt sein, solche Fälle hier kurz zu skizzieren, um so mehr, als die Tetanie hier in Stuttgart eine seltene Krankheit ist, so $\mathrm{da} \beta$ andernorts festgestellt werden muß, ob objektiv nachweisbare Überempfindlichkeit des Vestibularis bei darauf gerichteter Aufmerksamkeit wirklich so selten ist, wie man der Literatur nach annehmen muß.

Über den Zusammenhang von Ohrenleiden und Tetanie finden sich in der Literatur einige Hinweise, die zunächst kurz erwähnt seien. Der erste Fall wurde von Curschmann 1904 mitgeteilt (Otitis media mit kurz danach einsetzenden Tetaniekrämpfen). In einem zweiten Falle von Otitis media gelang es Hoffmann durch Ausspritzen des Ohres in der Latenzzeit Anfälle auszulösen. 1917 fanden 
Frey und Orzechowski, daß Tetanie gar nicht selten bei - Otosklerose vorkäme, also bei einer Krankheit, die wir als konstitutionell bedingt ansehen, wodurch diese Beobachtungen in Parallele mit dem Tetaniekatarakt rücken. Der Vestibularis scheint in den Fällen dieser Autoren nicht mitbetroffen gewesen zu sein. Ferner ist ein Fall von Curschmann zu nennen, der mit Menière vergesellschaftet war. Hoffmann sah in einem Fall von Tetanie Schwindelanfälle von 5-6 Minuten Dauer auftreten, wobei Unfähigkeit bestand, den linken Arm zu heben. Daß von Chvostck Übererregbarkeit des Akustikus beschrieben ist, sei ebenfalls bemerkt. Erwähnt sei schließlich noch, daß sich in der bekannten Monographic von Frankl-Hochwart über das. Ohr nur die folgende Angabe findet: „Über Anomalien der Gehörschärfe wird nicht geklagt, doch beschweren sich die Patienien nicht selten über. Ohrensausen." Und ähnlich äußert sich Phlebs, der folgendes anführt: „Von seiten des Gehörs wird vielfach über lästige Ohrengeräusche geklagt, doch konnte bisher im Zusammenhang mit der Tetanie niemals eine Abnahme der Hörschärfe nachgewiesen werden." Der Vestibulari; wird gar nicht erwähnt.

Ich möchte nunmehr hier die Krankengeschichte eines typischen Falles von Tetanie folgen lassen: Typische manifeste. Tetanie mit Flimmerskotomen (ohne Migräne), mit VestibularisÜberregbarkeit (ohne Gehorstörung und Schwindel), sowie mit leichter Parosmie und Parageusie.

Fall 11): August St., geb. 1896, Schneider. Sucht das Krankenhaus wegen Krampf auf. Der Krampf beginne mit einem dumpfen Gefühl in den Fingerspitzen. Dann ziehen sich die Finger langsam zusammen. Sie gehen in Pfötchenstellung. Die Krämpfe aszendieren dann, so daß auch die Arme betroffen werden. Dasselbe gelte von der unteren Extremität. Auch der Rumpf werde nicht verschont. Die Dauer der einzelnen Anfälle sei ganz verschieden, sie schwanke zwischen 5 Minuten und 2 Tagen (?). Während des Anfalls habe er Flimmerskotome, dagegen keinea Schwindel, kein Ohrensausen.

Als Kind habe er keine Anfälle gehabt, auch nicht beim Zahnen. Der 1. Anfall kam mit 17 Jahren, dann war er bis 1920 verschont.

Von früheren Krankheiten ist ein Lungenspitzenkatarrh au erwähnen und vor allem eine sehwere Rach itis, so daßer erst mit 6 Jahren laufen lernte.

Libido wenig entwickelt.

1) Dieser Fall wurde von Herrn Geh. Rat Sick im Ärteverein Stuttgart demonstriert. 
Status: Es handelt sich um einen kleinen Patienten von $155 \mathrm{~cm}$ Länge. Caput quadratum. Eingefallene Nasenwurzel. Die Augen stehen weit auseinander. Gesicht hat myxödematöses Aussehen, ohne daß aber eine teigige Schwellung bestände (Tetanie-Gesicht). Genu valgum Pedes plani. Hernia umbilicalis. Die Pubes sind von femininem Typ. Keine Achselhöhlenhaare. Auch sonst geringer Haarwuchs. Patient rasiert sich nur selten. Kopfhaar dicht. Hals gedrungen. Keine Struma. Schilddrüse palpabel.

Innere Organe ohne Befund (auch am Magen kein pathologischer Befund).

Gang ohne Befund, jedoch crmüdet Patient leicht.

Nervensystem: Pupillen reagieren auf Licht und Konvergenz prompt.

Die Augenbewegungen sind frei. Kein Nystagmus.

Augenhintergrund normal.

Lichtbrechende Medien o. B.

Facialis und Hypoglossus o. B. Jedoch Chvostek + .

Utber die übrigen Hirnnerven s. u.

Reflexe: Hautreflexe regelrecht.

Sehnenreflexe sehr lebhaft, an der unteren Extremität klonusartig.

Keine Pyramidensymptome. (Babinski usw. -)

Sensibilität: Abgesehen von den Parästhesien im Anfalle objektiv keine Abweichung von der Norm nachweisbar.

Trousseau: +++ .

Elektrische Untersuchung: Erb +++ . Akusticus elektrisch überempfindlich.

Trophische Störungen: Zähne geriffelt.

Nägel ohne Befund.

Geruch: verschiedene Stoffe werden nicht erkannt!

Geschmack: saure, salzige und bittere Speisen werden verwechselt, nicht dagegen süße, die stets erkannt werden, manchmal etwas zögernd. Die $\Lambda$ ngaben darüber sind bestimmt.

Gehör (Dr. Jahr): Cochlearis beiderseits intakt. Es besteht eine ausgesprochene Empfindlichkeit des Bogengangapparates gegen termische Reize. Der Ausschlag des Nystagmus horizontalis bei Kältespülung ist sehr stark.

Epikritisch möchte ich mich auf das folgende beschränken: Bei einem Patienten mit manifester Tetanic liegt cine schwere Rachitis vor, die zu leichtem Zwergwuchs führte. Daneben bestehen Zeichen für eine Störung der endokrinen Drüsen (Behaarung von femininem Typ, sowie Zeichen, die auf die Schilddrüse hinweisen: gedunsenes Aussehen des Gesichtes [s. o.] und Hernia umbilicalis.) Die Rachitis ist zum Abschluß gekommen zu einer Zeit, als noch keinerlei Krämpfe bei dem Patienten aufgetreten waren. Sie stellten sich erst viele Jahre 
später ein. Dauer und Schwere der Anfälle ist eine exorbitante. Während die Motilität im übrigen keine Besonderheiten bot, ebensowenig wie die Sensibilität (diese war sogar relativ wenig gestört), traten eine Reihe ungewöhnlicher sensorischer Erscheinungen auf. Zur Zeit der Anfälle kam es bei dem Patienten zu Flimmerskotomen, ohne daß Schwindel, Ohrengeräusche oder migräneartiger Kopfschmerz sich hinzu gesellten. Wir haben hier also in den Flimmerskotomen ein Zeichen der Übererregbarkeit des Nervus opticus vor uns, was ja bei der multilokulären Erregbarkeitssteigerung in Anlehnung an die von Curschmann beschriebene (und auch in unserem Falle beobachtete) Übererregbarkeit der übrigen censorischen Nerven nicht weiter erstaunlich ist. Soviel ich weiß, ist diese Übererregbarkeit des N. opticus bisher nicht beschrieben worden, von Curschmann allerdings theoretisch postuliert worden. Vielleicht gehört hierher ein Fall von Hoffmann, der weiter unten noch zu erwähnen sein wird. Die für gewöhnlich notierten Augenerscheinungen bei Tetanie sind: Krämpfe der Muskeln, Katarakt und Neuritiden, sogar mit Atrophien. Hier aber handelt es sich um Skotome, wi wir sie wohl bei der Migräne finden, während am Auge selbst ein objektiver pathologischer Befund fehlte, auch am Augenhintergrund, wie auch andererseits keine weiteren Anzeichen für Migräne vorlagen. Dies verdient um so mehr hervorgehoben zu werden, als ja Zusammenhänge zwischen der Epilepsie und der Tetanie bestehen wie auch andererseits zwischen der Epilepsie und der Migräne.

Auch Störungen des Geruchs und des Geschmacks sind bei unserem Patienten objektiv nachweisbar. Die Parosmie und Parageusie sind äußerst seltene Erscheinungen, auch wenn sie nicht sehr ausgeprägt sind.

Dazu kommen noch die Störungen des Vestibularis, über die in der Krankengeschichte bereits das -Notwendige gesagt ist. (Von der elektrischen Übererregbarkeit des Akusticus kann hier als häufig beobachtetes Symptom abgesehen werden.) Oben ist bereits hervorgehoben worden, daß bisher nur in einem Fall von Curschmann, der mit Menière kombiniert war, eine kalorische Übererregbarkeit nachgewiesen wurde. Hier aber liegen keinerlei Störungen von seiten des Ohres vor. Erst die darauf gerichtete Untersuchung ergab die äußerst starke kalorische Übererregbarkeit des Vestibularis bei sonst intaktem äußerm und innerm Ohr. Und der hier mitgeteilte Fall mit zahlreichen sensorischen Reizerscheinungen (Vestibularis-Übererregbarkeit, Parosmie, Parageusie und Flimmerskotome) tritt damit in Parallele zu dem von 
Curschmann geschilderten Fall mit sensorischen Erscheinungen, in denen die Parosmie und Parageusie allerdings auch in der Latenzzeit dominierten.

Schließlich sei noch erwähnt, daß anamnestisch keine Angaben eruiert wurden, die auf eine Spasmophilie in der Kindheit hinwiesen.

In einem 2. Falle, in dem ebenfalls eine Vestibularis-Überempfindlichkeit vorlag, bestand nur ein "tetanoider Symptomenkomplex", wie Frankl-Hochwart dieses von ihm beschriebene Syndrom genannt hat: Parästhesien, Ohvostek und Erb; aber kein Trousseau ${ }^{1}$ ). Etwas hänfiger als manifeste Tetanie sind zurzeit hier in Stuttgart solche Fälle, in denen nur eine mechanische und elektrische Übererregbarkeit vorhanden ist ohne Parästhesien oder Krämpfe. Mitunter kommt es allerdings zu uncharakteristischen Crampi, sei es, daß sie sich als Wadenmuskelkrämpfe äußern, sei es, daß sie als Schreiblkrämpfe imponieren. Sie werden nicht selten als Hysterie gedeutet, was um so bedauerlieher ist, als damit oft genug eine soziale Schädigung des Patienten verbunden ist und als andererseits kaum eine zweite Nervenkrankheit so leicht günstig beeinflußbar ist wie die Tetanie.

Ich lasse nunmehr Fall 2 folgen: Mit 3 Jahren Spasmophilie, in der Pubertät Epilepsie, jetzt (mit 21 Jahren) halbseitiger tetanoider Symptomenkomplex mit VestibularisÜbererregbarkeit.

Wilhelmine Fr., geb. 21. II. 1900, Stütze. Mutter wegen Dementia praecox in einer Irrenanstalt gewesen. Vater gesund. Patientin ist einziges Kind. Die Geburt war normal. Mit 3 Jahren, zur Zeit als die Backzähne kamen, bekam sie Krampfanfälle. Mit 13 oder 14 Jahren bekam sie nach Schwimmen nachts Anfälle; dabei soll sie Schaum vor dem Mund gehabt haben. Mit 19 Jahren hatte sie auf einem Ausflug an einem heißen Tage Anfälle, die sich in den nächsten 3-4 Tagen fast ununterbrochen wiederholten. Dabei wurde der ganze Körper ersehüttert. Der Mund war nach links verzogen. Zungenbiß. Patientin kam in eine Anstalt, wo Epilepsie diagnostiziert wurde. Ende des Jahres dort entlassen. Seitdem keine neuen Anfälle mehr. Sie sucht jetzt den Arzt wegen folgender Beschwerden auf: der linke Arm sei ihr oft ,schwach“", und es sei ihr mitunter so, als wenn ein elektrischer Schlag durch den linken Arm ginge; sie könne dann die Sachen in der Hand nicht halten. In den Fingern

1) Von dem ,tetanoiden Symptomenkomplex" wurde früher die latente Tetanie unterschieden, bei der es zum Auftreten von Krämpfen bei Auslösung des Trousseauschen Zeichens kam. Diese beiden Termini werden neuerdings aber promiscue gebraucht. 
der linken Hand, die in kaltem Wasser leicht abstürben, „Kribbeln“. Keine Wadenkrämpfe. Dagegen gibt sie nachträglich an,daB sie beim Schreiben in der Schule oft "Zittern" gehabt habe.

Von seiten der Sinnesorgane keinerlei Beschwerden, insbesondere keine Schwerhörigkeit und kein Ohrensausen. Dagegen systematischer horizontaler Schwindel. Menses mit 14 Jahren eingetreten, regelmäßig, schwach, 3-4 Tage dauernd. Kurz vor Eintreten der Periode werden die geschilderten Beschwerden schlimmer. Ob die Krämpfe mit 13, 14 Jahren im AnschluB oder kurz von der ersten Periode gewesen sind, weiß Patientin nicht.

Status: Es handelt sich um eine mittelgroße Patientin, an deren inneren Organen kein krankhafter Befund zu erheben ist. Auch finden sich keinerlei Degenerationsmerkmale.

Nervensystem: Pupillenreaktion prompt; Augenbewegung frei. Kein Nystagmus, kein Katarakt. Augenhintergrund o. B.

Facialis und Hypoglossus o. B. Jedoch Chvostek + .

Geruch, Geschmack o. 13.

Kalorischer Nystagmus vom Ohr aus sehr stark auslösbar mit Auftreten von heftigem Schwindel, so daß die Patientin längere $Z$ eit liegen muBte.

Reflexe: Hautreflexe regelrecht, Sehnenreflexe lebhaft.

Keine Pyramidensymptome.

Sensibilität: Objektiv keine Abweichung von der Norm nachweisbar; subjektiv Parästhesien.

Keine trophischen Störungen.

$\mathrm{Erb}+++$. Akusticus elektriseh überempfindlich.

Trousseau links + (sensibel; d.h. es treten starke Parästhesien, brennende Schmerzen, auf).

Hoffmann + .

Patientin bekam Chinin sulf. und Calcan. Zwei Monate später war Erb negativ, Chvostek negativ, während die elektrische Utbererregbarkeit des Akusticus noch bestand, wie auch links der sensible Trousseau. Während der Periode wird Calcan nicht gut vertragen.

Epikrise: Eine Patientin, die bei Durchbruch der Backzähne Krämpfe bekam, hatte später Crampi, mit 14 Jahren und mit 19 Jahren. Der Charakter der letzteren wurde von autoritativer Seite bei mehrmonatlicher Spitalbehandlung als Epilepsie festgestellt. Auf die Koinzidenz von Fpilepsie und Tetanic werde ich weiter unten eingehen, ebenso auf das Rezidivieren der Krämpfe zur Zeit der Menarche. Bei späteren Untersuchungen, die wegen rnotorischer Störungen des Armes usw. vorgenommen wurden (beim Anfassen von Gegenständen wird die linke Hand steif; außerdem links Parästhesien) wurden beiderseits 
Erb und Chvostek festgestellt, womit die Zugehörigkeit des Krank heitsbildes trotz des Fehlens von typischen Tetaniekrämpfen zum tetanoiden Symptomenkomplex erwiesen ist. Zeichen von Rachitis fehlen, ebenso solche von seiten des Ausfalls der Schild-. drüse.

Auch in diesem Fall bestand wie in Fall 1 eine starke kalorische Übererregbarkeit des Vestibularis, so daß Patientin nach der kalorischen Prüfung längere Zeit liegen mußte. Von seiten der sensorischen Nerven liegt sonst keinerlei Störung vor. Ich gehe darauf nicht weiter ein, sondern verweise auf die Epikrise von Fall 1.

Bei Prüfung des Trousseauschen Phänomens, das hier negativ ausfiel, klagte die Patientin schon nach sehr kurzer Zeit am linken Arm über starke Parästhesien, obgleich die Binde nicht fester angelegt war als rechts, wo keine abnorme sensible Überempfindlichkeit bestand. Ich habe dasselbe noch in einem zweiten Fall, in dem Trousseau ebenfalls negativ war, gefunden und möchte es daher nicht als zufällig ansehen, sondern mit dem von Hoffmann angegebenen Zeichen der elektrisehen Übererregbarkeit sensibler Nerven in Parallele stellen. Ich habe es in der Krankengeschichte als "Trousseau + sensibel" bezeichnet.

Die „Steifigkeit“, sowie die Parästhesien sind bei unserer Patientin halbseitig. Halbseitigkeit der Krämpfe ist extrem selten. Eine Erklärung für ihr Vorkommen läßt sich nicht geben, da wir ja in der Tetanie eine allgemeine Intoxikation sehen. Immerhin ist die Halbseitigkeit nicht ohne Analogien. So beobachtete ich einen Fall von Encephalitis arsenicosa (nach Salvarsan), bei dem sich bei der Sektion verstreute Herde in beiden Gehirnhälften fanden. Dabei waren 1-2 Tage vor dem Exitus eigentümliche Krämpfe nur im linken Arm aufgetreten, wobei die Hand in Tetaniestellung ging. Auch an die Hemikranie sei erinnert, bei der es ja auch unerklärlich ist, warum der Kopfschmerz in der Regel einseitig ist. In dem erwähnten Falle von Encephalitis arsenicosa wurde ebenso wie bei unserem Falle von latenter Tetanie (wie auch bei den Fällen der Literatur) nichts gefunden, was die Halbseitigkeit erklären könnte. Ganz besonders merkwürdig ist dabei, daß viele Fälle von Hemitetanie, wie auch der unsrige, mit allgemeinen epileptischen Krämpfen vergesellschaftet sind. Eine Bevorzugung einer Seite besteht nicht. Auch Überanstrengung, Mehrgebrauch usw. scheinen keine Rolle zu spielen. Ich lasse kurz die Fälle von Hemitetanie folgen: 
wurde ein "tetanoider Symtomenkomplex" festgestellt. In Fall 3 traten gleichzeitig mit der Menarche Krämpfe auf, die die Patientin als „Wadenkrämpfe“ unđ „Schreibkrämpfe“ bezeichnete, die mit Parästhesien verbunden waren und die durch die charakteristischen Signa (Erb, Chvostek) sich als Tetanie manifestierten. Gleichzeitig oder wenig später kam es dabei zu schweren Anfällen allgemeiner $\mathrm{Na-}$ tur, die mit Bewußtseinsverlust einhergingen. An der Diagnose Epilepsie dürfte daher kein Zweifel sein. Hinzu kommt nun noch die Ohrenschädigung, so daß vom Facharzte die Diågnose ,Menière ?" gestellt wurde. Die Häufung der Krampfneurosen ist aber noch größer. Aus den zablreichen Symptomen läßt sich noch ein Komplex herausschälen: Flimmerskotome, Kopfschmerzen, Nausea, d. h. es bestand noch eine Migräne. Die Kombination von Tetanie mit Menière ist, wie schon oben gesagt, bereits von Curschmann beschrieben worden. Eine Hemikrania lag vielleicht in einem Falle von Hoffmann vor. Übrigens gewinnt unser Fall 3 dadurch Beziehungen zu Fall 1, bei dem es während des Anfalls zu Flimmerskotom kam.

Die Beziehungen zwischen Tetanie und Epilepsie sind kürzlich von Redlich ausführlich abgehandelt worden, so daß darauf nicht näher einzugehen ist. Trotzdem besteht noch immer eine fast unüberbrïckbare Differenz in der Grundfrage, ob ein ursächlicher Zusammenhang besteht oder ob es sich um die zufällige Koinzidenz zweier nicht seltener Krankheiten handele. Gerade Fälle, wie die mitgeteilen, in denen auch Fäden zu andern Neurosen ziehen, zeigen meines Erachtens, daß es näher liegt, einen kausalen Zusammenhang anzunehmen. Die schärfste Ablehnung hat diese Auffassung von Thiemich und Birk, sowie von Bolten erfahren. Die genannten Pädiater weigern sich sogar in der Spasmophilie eine Disposition für die Epilepsie zu sehen. Verlangt Birk, daß bei Epilepsie alle anamnestisch zu erhebenden Crampi der Kindheit als Epilepsie zu werten seien, so sieht ähnlich Bolten die Kindheitskrämpfe in. solchen Fällen ,als vermutlich cerebral“ bedingt an, als ein „Symptom organischer Gehirnläsion“. Meines Erachtens ist diese Anschauung durch nichts erhärtet. Fälle, in denen es gelegentlich (z. B. bei Zahnen) zu Krämpfen kommt und in denen es dann erst nach vielen Jahren, etwa in der Pubertät, zum Ausbruch einer Epilepsie kommt, lassen wohl eine organische Läsion als Grundlage ausschließen, da man hierbei einen mehr kontinuierlichen Fortgang bis zum Status epilepticus erwarten müßte. Ein (nicht postoperativer) Fall von Ernst Freund (als Beispiel aus den Fällen der Literatur entnommen) spricht 
auch im ausgeführten Sinne: Eine Frau bekam während der Laktation eine Tetanie, 1 Jahr später, wiederum während der Laktation, trat die Tetanie von neuem auf, dieses Mal vergesellschaftet mit Epilepsie. Wie erzwungen die Annahme von Birk ist, geht daraus hervor, daß er für Anfälle in der Kindheit die Zugehörigkeit zur Spasmophilie auch dann bezweifelt, wenn nicht der ganze Symptomenkomplex vorliege: „Ein oder das andere spasmophile Symptom wird gelegentlich auch einmal bei epileptischen Kindern gefunden", wobei er, wie zu beachten ist, bei solchen Kindern Anfälle, die nicht den ganzen Symptomenkomplex aufweisen, eben als epileptisch auffaßt. In unserm 2. Falle, z. B., wo die latente Tetanie nur durch darauf gerichtete Untersuchung entdeckt wurde, während sie wohl schon lange bestanden haben dürfte, spricht doch alles dagegen, daß die Krämpfe mit 3 Jahren schon epileptische waren. Gerade der Zusammenhang mit der Dentition läßt sie mit großer Wahrscheinlichkeit als spasmophil erscheinen.

Das Fehlen sämtlicher thyreogener Symptome in Fall 2 und 3, die man als cerebral bedingte Epilepsie auch nicht wird auffassen wollen, spricht auch gegen ein weiteres Postulat von Bolten, wonach die Epilepsie ,auf einem stark defekten Stoffwechsel infolge von Hypothyreoidie (und Hypoparathyreoidie) beruht". Solche Fälle von Tetanie und Epilepsie, bei denen nichts für eine organische Erkrankung des Gehirns spricht und bei denen andererseits auch alle klinischen Zeichen von Hypothyreoidie fehlen (analoge Fälle sind auch von anderer Seite mitgeteilt worden) sprechen wohl dafür, daß eine andere endokrine Drüse, wahrscheinlich die Nebenschilddrüsen allein dysfunktionieren, und daß diese Dysfunktion die Auslösung der Epi- und der Tetanieanfälle verursacht, $d . h$. daß der Mechanismus derselbe ist wie bei den postoperativen Fällen.

Gewiß gibt es (vereinzelt) Fälle von Tetanie und Epilepsie, wo das Zusammentreffen ein zufälliges ist. Wenn aber dieselbe endogene oder exogene Noxe beide Krankheiten zum Ausbruch bringt, so wird man wohl annehmen, daß beide auf demselben Wege zustande kommen. (Vgl. hierzu bes. Curschmann.)

Man muß auch Bolten weiter widersprechen, wenn er die cerebral bedingte Tetanie nicht nur als nicht exwiesen, sondern sie sogar als unwahrscheinlich ansieht. Damit wird den Tatsachen Zwang auferlegt. Die Zalil der Tetaniefälle bei Läsion des Gehirns ist zu groß, aìs daß man hierin eine zufällige Koinzidenz erblicken könne. Es sei an die Befunde von Pick erinnert, der bei Tetanie in den feineren und 
feinsten Gefäßen Verkalkung nachwies, an Curschmanns Beobachtung über das gleichzeitige Vorkommen mit Pseudobulbärparalyse, ferner an den von Exdheim und Frankl-Hochwart beschriebenen Fall von Tetanie bei zystischem Tumor des Kleinhirns, wie ja überhaupt tetanoide Krämpfe bei Affektionen des Kleinhirns des öfteren beschrieben worden sind. Auch bei Erkrankung der subkortikalen Ganglien sind ähnliche Beobachtungen gemacht worden, aber auch bei Erkrankung der Hirnrinde. So sah ich einen Fall von Streifschußverletzung des Kopfes mit kortikaler Lähmung, die allmählich zurückging. Gleichzeitig bestand eine Epilepsie. Neben diesen Anfällen kam es nun noch zu andern Krämpfen (ebenfalls erst seit der Verwundung), bei denen athetoide Bewegungen auftraten, sowie tetanische Krämpfe der früher gelähmten Hand. Auf Grund solcher Beobachtungen kann man sich wohl Spiegel anschließen, daß die Tetanie nicht nur ein günstiges Terrain für die Epilepsie darstelle, sondern daß auch andererseits Veränderungen des Großhirns begünstigend für das Entstehen von Tetanie sei.

Jedenfalls kann man sagen, daß die Tetanie und die Epilepsie sehr nahe verwandte Krankheiten sind, die ineinander übergehen können oder auch auf Grund derselben Noxe (nicht nur postoperativ) entstehen. Unser letzter Fall, der vier Neurosen nébeneinander zeigt, läßt die nahe Verwandtschaft dieser Syndrome besonders schön erkennen. Daß die Migräne der Epilepsie nahesteht, ist ja bekannt, ebenso daß der Menièresche Symptomenkomplex als Aura des epileptischen Anfalls vorkommt. Daß das Vorkommen dieser ebengenannten drei Krampfneurosen nur durch eine gemeinsame Ursache erklärt werden kann, liegt auf der Hand. Die gemeinsame Ursache liegt in einer Dysfunktion des endokrinen Drüsensystems, die sich in diesem Falle (klinisch greifbar) im Infantilismus des Uterus, in den Menstruationsstörungen äußert und nach unseren Anschauungen über das endokrine System auch auf die andern Blutdrüsen zurückwirkt.

Zum Schluß ist noch die Frage zu erörtern, wie es kommt, daß die Tetanie häufiger als früher in Stuttgart auftritt. Dabei ist noch zu bemerken, daß auch die postoperativen Fälle sich vermehrt haben, wie Sick in einem Vortrage im Ärzteverein betonte. Daß dabei der Genius loci eine Rolle spielt, wurde in Friedenszeiten als sicher angenommen. Hatte doch z. B. Kocher in Bern unter 3000 Kropfoperationen 5 Fälle von Tetanie, dagegen v. Eiselsberg in Wien unter 
356 Operationen 16 Fälle. Wenn also die Tetanie hier zugenommen hat (wie ja übrigens auch in andern deutschen Städten, z. B. Hamburg), so wird man natürlich die Ursache in Veränderungen suchen, die der Krieg gebracht hat, also in erster Linie in der Kriegsnahrung. Dabei erhebt sich aber gleich wieder die Frage, ob die Tetanie wirklich nur in den klassischen Städten gehäuft vorkommt oder ob sie nicht latent mehr oder weniger überall anzutreffen ist. Aufschluß darüber gibt das Chrosteksche Zeichen. Folgt man S. Schönborn, ,auch die leichten Grade als Symptome des im übrigen latenten Syndroms aufzufassen, das wir Tetanie nennen", so kann man sich unschwer vorstellen, daß eine neue Noxe (Krieg) die Verschlimmerung bis zum tetanoiden Symptomenkomplex bringt oder gar, was allerdings selten zu sein scheint, bis zur manifesten Tetanie. Man braucht dann nur weiter mit Schönborn anzunehmen, daß das Chvosteksche Zeichen in sog. tetaniefreien Städten zeige, ,daß es dort - vielleicht auf Grund noch unbekannter klimatischer Verhältnisse - eben nicht zu den schweren Tetanieformen kommt; und auch das lange Persistieren wäre einfach als das Vorkommen chronisch leichtester Tetanieformen aufzufassen". Spricht Schönborn hier von klimatischen Verhältnissen, so wird man auf Grund der Kriegserfahrungen wohl lieber konstitutionelle Momente der Bevölkerung zur Erklärung heranziehen. Auf jeden Fall wird es so leichter sein, das gehäufte Auftreten unserem Verständnis näher zu bringen, als wenn man keine Vorposten annähme.

Eine zweite Krankheit, die zweifellos seit dem Kriege viel häufiger zur Beobachtung kommt, sind die Arthropathien. Nach Veröffentlichungen aus verschiedenen deutschen Städten findet man diese beiden Krankheiten neuerdings oft gehäuft bei denselben Personen, während friiher (anders Krajewska, der $48 \mathrm{mal}$ im Laufe von 10 Jahren puerperale Tetanie mit Osteomalacie sah!) das gemeinsame Vorkommen äuBerst selten war (Curschmann, Ebstein, Freund, Schüller u. a.; in neuerer Zeit u. a. Alwens, Bittorf, Schlesinger, Sauer. In der Arbeit des letztgenannten Autors Literatur.) Auch bei Fragilitas ossium congenita sah Bolten diese Kombination, wobei wichtig ist, daß er bei einigen Familienmitgliedern ohne Knochenveränderung auch Tetaniezeichen fand. In den Fällen von Kriegsknochenerkrankung, die ich selbst untersuchen konnte, fand ich niemals Tetaniezeichen, ohne allerdings in allen Fällen darauf geachtet zu haben. Die Fälle von Tetanie betrafen nun wie gewöhnlich nur oder fast nur Leute der ärmeren Schichten (ist ja die Tetanie eine „Arme-Leute-Krankheit“), während 
Arthropathien auch bei reicheren Personen beobachtet werden. Ich glaube daher, daß es sich entweder um zwei verschiedene Noxen handelt oder daß dieselbe Noxe zwei verschiedene Systeme beeinflußt. Für die Tetanie, glaube ich, geben diese Beobachtungen eine Stütze der Fuchsschen Ergotinhypothese. Fuchs wies bekanntlich auf die gemeinsamen Züge des Ergotismus convulsivus und der Tetanie hin und nahm an, daß das dem billigen Mehl beigemischte Mutterkorn die Ursache der Tetanie sei, eine Anschauung, die Oppenheim, Bolten u. a. mehr oder minder anerkennen. Diese Hypothese ist aber andererseits bis in neuere Zeit auch bekämpft wurden. Von den Gegnern sei nur W. Goldschmidt genannt, der in der Gefangenschaft Gelegenheit hatte, Fälle von Erkrankung an Ergotismus zu sehen, und der nur selten versucht war, Tetanie zu diagnostizieren. Folgt man der Fuchsschen Anschauung, so läßt sich das gehäufte Vorkommen der latenten Tetanie (bei disponierten Menschen) etwa so erklären: die ungeübten, überarbeiteten Landarbeiter entfernten das Mutterkorn nicht mehr so sorgsam wie dies früher geschah. Gerade das Kriegsbrot wurde aber von vermögenderen Leuten, dauernd oder zeitweilig, auf alle Arten zu vermeiden gesucht. Ein Mangel an Kalzium oder an Rachitisvitaminen bei unseren Fällen anzunehmen, scheint mir wegen der mangelnden Koinzidenz der beiden genannten Krankheitsbilder nicht angängig zu sein.

\section{Literatur.}

Birk, Über die Anfänge der kindl. Tetanie. Ergebn.d.inneren Med. u. Kinderh. 1919, Bd. 3. S. 551.

Boenheim, Pseudosklerose. Zeitschr. ges. Neurol. u. Psych. 1920, Bd. 60, S. 10. Bolten, Epilepsie und Tetanie. Deutsche Zeitschr. f. Nervenheilk. 1917, Bd. 57, S. 160. - Ứber Hyp sthyreoidie. Deutsche Zeitschr. f. Nervenheilk. 1917, Bd. 57. S. 119.

Curschmann, Tetanie, Pseudotetanie usw. Deutsche Zeitschr. f. Nervenheilk. 1904, Bd. 29, S. 239. - Über einige ungew. Ursachen und Syndrome der Tetanie. Deutsche Zeitschr. f. Nervenheilk. 1910, Bd. 39, S. 36. - Cerebrale Symptome der Tetanie usw. Deutsche Zeitschr. f. Nervenheilk. 1912, Bd. 45. - Epilepsie und Tetanie. Deutsche Zeitschrift f. Nervenheilk. 1918, Bd. 61, S. 1. - Úbsr otogene Auslösung von Tetanie-Anfällen. Wiener klin. Wochenschr. 1918, Nr. 19. - Über sensible und sensorische Tetanie. Münchn. med. Wochenschr. 1919, S. 983.

Ebstein, Ungew. Syndrome der Tetanie usw. Med. Klinik 1911, S. 1496.

Falta und Kahn, Klinische Studien über Tetanie usw. Zeitschr. f. klin. Med. 1912 , Bd. 74 , S. 108. 
Frank1-Hoohwart, Die Tetanie der Erwashsenen. 2. Aufl. Wien-Leipzig 1907.

Ernst Fre und, Beziehungen der Tetanie zur Epilepsie usw. Arch. f. klin. Med. 1903, Bd. 76, S. 10.

Heinr. Freund, Zwei seltene Fälle von Tetanie. Wiener med. Wochenschr. 1899, S. 1788 .

Frey und Orzechowski, Über das Vorkommen latenter Tetanie bei OtosIerose. Wiener klin. Wochenschr. 1917, Nr. 32-34.

Friedberg, Parmakol. Prüfung der vegetat. Nervensystoms im Kindesalter. Arch. f. Kinderheilk. 1921, Bd. 69, S. 107.

Alfred Fuchs, Analog. im Krankheitsbild des Ergotismus und der Tetanie. Wiener med. Wochenschr. 1911, S. 1853.

W. Goldschmidt, Ergotismus und Tetanie. Wiener klin. Wochenschr. 1919. Grahe, Zwei eigenartige Fälle von Tetanie. Med. Klinik 1914, S. 1221.

Hoffmann, Beiträge zur Lehre von der Tetanie. Deatsche Zeitschr. f. Nervenheilk. 1897, Bd. 9, S. 278.

Krupičkovă, Bemerkenswerter Fall von Hemitetanie. Ref. Wiener med. Wochenschr. 1919, S. 1341.

Peter Müller. Zitiert nach Kehrer, Geburtsh..gynäkol. Bedeutung der Tetanie. Arch. f. Gynäkol. 1913, Bd. 99, S. 372.

Munroe. Zitiert nach Bolten.

Oppler, Beiträge zur Kenntnis der Tetanie. Arch. f.klin. Med. 1887, Bd. 40, S. 232 .

Peritz, Spasmophilie der Erwachsenen. Zeitschr.f. klin. Med. 1913, Bd.77, S.190. Phlebs, Die Tetanie. Im Handb. der Neurologie von Lewandowsky, Bd. 4, S. 159, Berlin 1913.

Pick, Vorläufige Mitteilungen zur Pathologie derTetanie. Neurol.Zentralbl.1902, S. 578. - Weiterer Beitrag zur Pathologie der Tetanie. Neurcl.Zentralhl. 1903, S. 751 .

Redlich, Tetanie und Epilepsie. Monatsschr.f. Psych. u. Neurol. 1911, Bd. 30, S. 439 .

Sauer, Über Spätrachitis und ihre Beziehung zu den endokr. Drüsen. Deutsche Zeitschr. f. Chir. 1921, Bd. 162, S. 439.

Schönborn, Klinik zur menschlichen Tetanie. Deutsche Zeitschr. f. Nervenheilk. 1910, Bd. 40, S. 319.

Spiegel, Hemitetanie bei Großhirnläsion. Deutsche Zeitschr. f. Nervenheilk. 1920 , Bd. 65 , S. 310 . 